\title{
Successful Pregnancy Outcomes After Laparoscopic Management of Pheochromocytoma
}

\author{
Pradeep Prakash, MCh, Vidushi Kulshrestha, MD, ${ }^{2}$ Rashmi Ramachandran, MD, ${ }^{3}$ and Rajeev Kumar, $\mathrm{MCh}^{1}$
}

\begin{abstract}
Background: Pheochromocytoma is a rare cause of hypertension in pregnancy, which is often overlooked; especially in late pregnancy because of more prevalent pre-eclampsia. It has been associated with significant morbidity and mortality rates in both mother and fetus, if not diagnosed and treated in time. Minimally invasive surgery has been infrequently used for surgical management of pheochromocytoma in pregnancy, with $<20$ reported cases in English literature.

Case Presentation: A 26-year-old pregnant woman presented at 9 weeks of gestation with complaints of palpitations, sweating, and headache; with past history of first trimester spontaneous abortion caused by accelerated hypertension. She was found to have hypertension and diabetes, but no pedal edema, weight gain, or proteinuria. Ultrasonogram and MRI of abdomen revealed a left adrenal mass and 24 hours urinary catecholamines levels were increased, suggesting a pheochromocytoma. After preoperative optimization in consultations with obstetricians, endocrinologists, and anesthetists, she underwent laparoscopic left adrenalectomy during 15th week of gestation. Perioperative hospital course was uneventful for both mother and the fetus. After adrenalectomy, her diabetes was cured and hypertension was controlled with single antihypertensive. She was readmitted at 31 weeks of gestation with accelerated hypertension and underwent emergency caesarean for impending eclampsia at 32 weeks, and delivered a healthy female baby. 131I-meta-iodobenzylguanidine (MIBG) scan and 68Ga-[1,4,7,10-tetraazacyclododecane-1,4,7,10-tetraacetic acid]-1-NaI(3)-octreotide positron emission tomography-CT (68Ga-DOTANOC PET-CT) scan was obtained in postpartum period to rule out any extra-adrenal pheochromocytoma, both of which did not reveal any abnormality. At 1 year follow-up, she is normoglycemic and hypertension controlled on single antihypertensive.

Conclusion: Pheochromocytoma in pregnancy is a rare but potentially lethal condition, and high index of suspicion is required for early diagnosis. Multidisciplinary coordination is required for effective management of this rare condition. Laparoscopic adrenalectomy is safe in second trimester of pregnancy for both mother and fetus.
\end{abstract}

Keywords: pheochromocytoma, pregnancy, laparoscopy, adrenalectomy

\section{Introduction and Background}

$\mathbf{P}$ HEOCHROMOCYTOMA IS A RARE tumor and it is even rarer in pregnancy. It is one of the important causes of hypertension in pregnancy; however, it is often overlooked owing to the more prevalent diagnosis of pre-eclampsia, especially in late pregnancy. It can lead to devastating complication to both mother and fetus if not treated in time, with highest reported complication rate during labor. Laparoscopy has been infrequently used for surgical management of pheochromocytoma in pregnancy, with $<20$ reported cases in English literature. We present the challenges faced during management of a case of pheochromocytoma in early pregnancy and effective outcomes using laparoscopic adrenalectomy.

\section{Presentation of Case}

A 26-year-old woman, diagnosed with hypertension 4 years earlier, presented at 9 weeks period of gestation in her second pregnancy, with complaints of palpitations, sweating, and headache. During her previous pregnancy, which ended in fetal loss at 12 weeks of gestation, she was diagnosed with accelerated hypertension. The measured blood pressures at our hospital were also $>160 / 100 \mathrm{~mm} \mathrm{Hg}$. There was no history of weight gain or pedal edema on examination. She was simultaneously diagnosed to have diabetes mellitus on admission, with random blood sugar $289 \mathrm{mg} / \mathrm{dL}$. She was started on $200 \mathrm{mg}$ labetalol and $10 \mathrm{mg}$ nifedipine, thrice daily each, for blood pressure control and subcutaneous insulin for glycemic control.

Departments of ${ }^{1}$ Urology, ${ }^{2}$ Obstetrics and Gynaecology, and ${ }^{3}$ Anaesthesiology, All India Institute of Medical Sciences, New Delhi, India. 

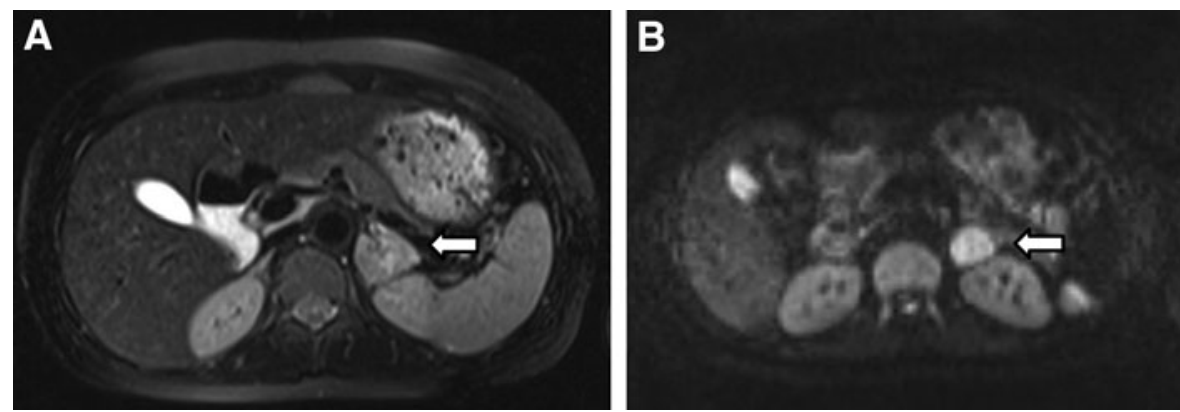

FIG. 1. MRI showing hyperintense lesion in left adrenal gland (white arrow) (A) T2 weighted image; (B) diffusion weighted image.
Routine blood investigations, including platelet counts and liver function tests, were normal. Urine analysis revealed daily protein excretion $<300 \mathrm{mg} /$ day. Ultrasonogram abdomen showed $4 \times 2.6 \times 2.9 \mathrm{~cm}$ left adrenal tumor and a single live intrauterine fetus. MRI abdomen revealed a T2 hyperintense mass involving left adrenal measuring $4 \times 3 \times 2.9 \mathrm{~cm}$ (Fig. 1). Urinary noradrenaline level was $577.5 \mu \mathrm{g}$ in a 24 hours sample (normal $0-90 \mu \mathrm{g} / 24$ hours), adrenaline was $14 \mu \mathrm{g} / 24$ hours (normal $0-20 \mu \mathrm{g} / 24$ hours) and vanillylmandelic acid was $17.5 \mathrm{mg} / 24$ hours (normal $0-15 \mathrm{mg} / 24$ hours). Hence the diagnosis of left pheochromocytoma was made.

Prazosin was added to her antihypertensive regime once a diagnosis of pheochromocytoma was established. She was advised oral salt and fluid replenishment. There were a number of issues that needed decision in her management. In view of the pregnancy, functional imaging such as 131Imeta-iodobenzylguanidine (MIBG) scan could not be performed to identify other adrenal or extra-adrenal tumors. Second, this was a precious pregnancy and there were high chances of fetal demise if pregnancy was continued in presence of a pheochromocytoma. Third was the timing and approach for surgery, open or laparoscopy.

These issues were discussed in a multidisciplinary meeting comprising teams from obstetrics, anesthesia, endocrinology, and urology, and the patient was counseled regarding the risks of continuance of pregnancy. She chose in favor of continuation of pregnancy and early adrenalectomy. It was thus decided to perform a mid-second trimester, laparoscopic adrenalectomy at 15 th week of gestation.

The antihypertensives and insulin were continued and prazosin was up-titrated to a final dose of $4 \mathrm{mg} /$ day in four divided doses at which adequate control of symptoms and blood pressure was achieved. She was given hydroxyprogesterone injection 1 day before surgery to prevent preterm labor. During surgery, an arterial line was established for continuous blood pressure monitoring. Central venous access was accomplished through her right jugular vein for administration of liquids and vasoactive agents. Serial arterial blood gases and glucose were monitored to prevent maternal acidosis and hypoglycemia.

General anesthesia was maintained with sevoflurane in air. Intraoperative hypertensive spikes were managed with titrated nitroglycerine infusion. She underwent laparoscopic transperitoneal left adrenalectomy with some modifications of the technique. She was placed in modified flank position; $70^{\circ}$ oblique to the horizontal. Pneumoperitoneum was made by open method to avoid injury to intra-abdominal organs. Three ports were placed to achieve adequate triangulation (Fig. 2). The whole procedure was carried out at lower intraabdominal pressure of $12 \mathrm{~mm} \mathrm{Hg}$.
The descending colon was mobilized along the line of Toldt and retracted medially. The left adrenal vein was identified at the cranial end of the left renal vein and was clipped using Hem-o-lock ${ }^{\circledR}$ clips (Fig. 3). After division of the adrenal vein, the tumor was dissected from the pancreas, spleen, and upper pole of left kidney using an ultrasonic dissector (Harmonic ${ }^{\circledR}$ scalpel; Ethicon Endosurgery, NJ). The operative time was 90 minutes and blood loss was $100 \mathrm{~mL}$. Surgery was uneventful and fetal viability was confirmed by ultrasonography obtained in the evening of surgery and on second postoperative day. She did not require any inotropic support in the postoperative period.

Oral progesterone tablets were continued after the surgery. She was discharged on seventh postoperative day on a single antihypertensive (labetalol) to control the hypertension. She was normoglycemic after surgery. Histopathologic examination of resected specimen confirmed a pheochromocytoma.

She continued regular antenatal visits till 31 weeks when she was again admitted for uncontrolled hypertension. Hypertension was managed with multiple antihypertensives and antenatal steroids were given for fetal lung maturation. She was managed conservatively till 32 weeks when her blood pressure was recorded to be $180 / 130 \mathrm{~mm} \mathrm{Hg}$ and started having mild headache. With a provisional diagnosis of impending eclampsia, injectable antihypertensive were started and injection magnesium sulfate was given. An emergency lower segment caesarean section was undertaken at 32 weeks in view of impending eclampsia and a live female baby weighing $2.5 \mathrm{~kg}$ was delivered. Six weeks after delivery, a complete evaluation for extra-adrenal pheochromocytoma was undertaken, including functional imaging studies. Her

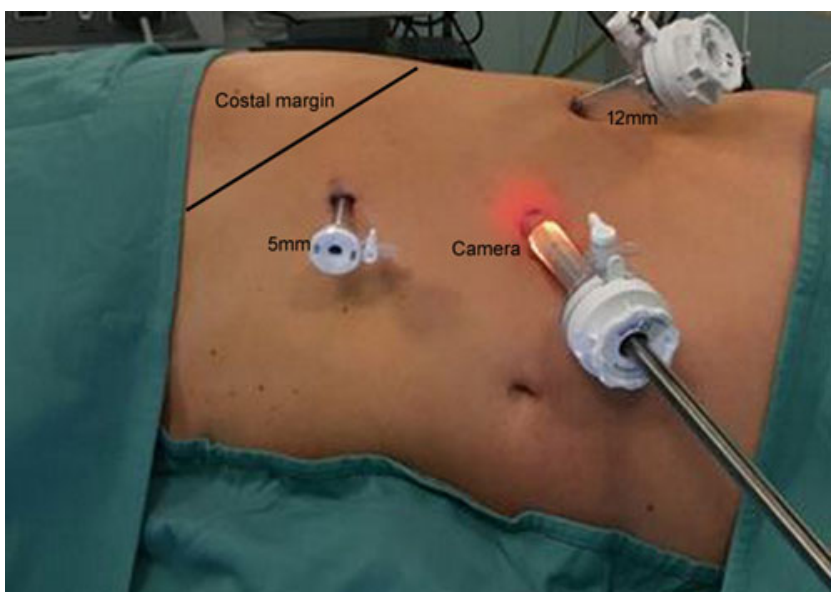

FIG. 2. Ports position. 
FIG. 3. (A) Intraoperative view of left adrenal vein (small white arrow), left adrenal tumor (large white arrow), and left renal vein (black asterisk); (B) left adrenal vein ligation using Hem-o-lock ${ }^{\circledR}$ clips.
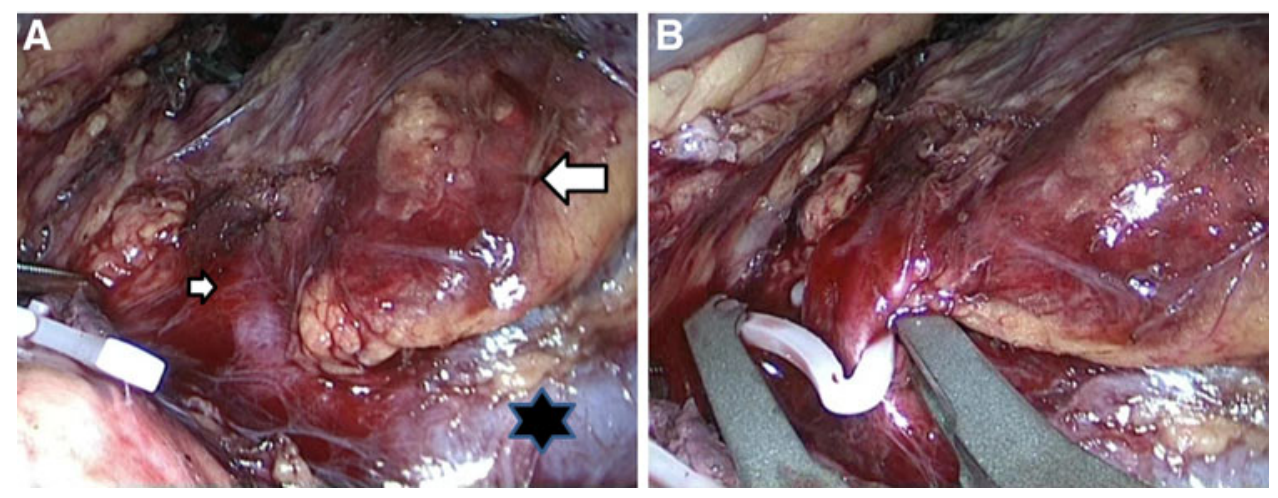

24-hour urinary catecholamines were normal. MIBG scan and 68Ga-[1,4,7,10-tetraazacyclododecane-1,4,7,10-tetraacetic acid]-1-NaI(3)-octreotide positron emission tomographyCT (DOTANOC PET-CT) were obtained, which did not reveal abnormal uptake anywhere in the body. She remained hypertensive at the time of discharge from the hospital, which was under control with single oral antihypertensive.

After 1 year of follow-up, the patient was normoglycemic; however, she remained hypertensive, controlled on single oral antihypertensive. MIBG scan and DOTANOC PET obtained again after 1 year did not reveal any evidence of extraadrenal paraganglioma. Her child was healthy and attained normal developmental milestones at the age of 1 year.

\section{Discussion}

Pheochromocytoma is one of the most threatening medical conditions in pregnancy. Historically, if undiagnosed and untreated, it resulted in maternal and fetal mortality rates in up to $10 \%-12 \%$ and $40 \%-50 \%$, respectively. However, with better diagnostic methods available, presently antenatal diagnosis is made in up to $83 \%$ of cases and this resulted in reduction of maternal mortality rate to $0 \%-2 \%$ and fetal mortality rate to $\sim 11 \%{ }^{1}$

The risks to the mother are caused by hypertensive crisis, and the risk is highest in peripartum period because of labor, anesthesia, delivery, and medications. The potential risks to fetus are caused by transient excessive maternal catecholamines, which can cause severe vasoconstriction in the placental circulation, which may result in placental abruption and intrauterine hypoxia.

Even with the advances in diagnosis, $15 \%-20 \%$ of cases remain unrecognized mainly because of more prevalent diagnosis of pre-eclampsia. However, a vigilant clinician can use certain differentiating features to suspect it. Preeclampsia tends to occur after 20 weeks of pregnancy, whereas pheochromocytoma may present anytime during pregnancy. Also proteinuria is an important component of pre-eclampsia, which is not seen in pheochromocytoma.

Surgical management and timing is controversial for pheochromocytoma in pregnancy. Second trimester is the ideal time for surgical treatment as organogenesis is complete and the endometrium is stable to withstand effect of anesthesia and surgery. First trimester intervention is associated high chances of miscarriage, whereas in third trimester abdominal exploration and access is difficult, and there is risk of premature labor. Before 28 weeks preferred approach is preoperative $\alpha$-blockade followed by adrenalectomy, whereas after 28 weeks it is safer to manage the patient conservatively till term and adrenalectomy at the time of elective caesarean. $^{2}$

Laparoscopic adrenalectomy has been underutilized in pregnancy because of assumption that pneumoperitoneum is associated with more complications. Also, with aim to minimize operative time, and thus to minimize intraoperative hemodynamic disturbances, many centers prefer open surgery. A few $(<20)$ cases of antenatal laparoscopic adrenalectomy are reported in English literature; earliest at 13th week of gestation by Pace and colleagues ${ }^{3}$ and latest at 29th week by Donatini et al. ${ }^{4}$

With growing experience, laparoscopic adrenalectomy is now preferred treatment in the second trimester. Various maneuvers to minimize risk include using open Hasson technique to gain access, keeping insufflation pressure preferably $<12 \mathrm{~mm} \mathrm{Hg}$, and monitoring fetal heart rate and maternal arterial blood gases. Meticulous preoperative preparation and intensive maternal and fetal monitoring during and after the procedure is essential to achieve optimal outcomes.

\section{Conclusions}

Pheochromocytoma in pregnancy is a rare but potentially lethal condition, for both fetus and the mother and high index of suspicion is required for diagnosis, especially in late pregnancy. Multidisciplinary coordination among obstetricians, endocrinologist, anesthetist, and surgeon is required for effective management of this rare condition. Adequate preoperative $\alpha$-blockade and $\beta$-blockade, if required because of tachycardia, is mandatory for safe removal of these tumors. Laparoscopic adrenalectomy is underutilized, but safe in second trimester for both mother and fetus.

\section{Disclosure Statement}

No competing financial interests exist.

\section{Funding Information}

No funding was received for this article.

\section{References}

1. Lenders WM. Pheochromocytoma and pregnancy: A deceptive connection. Eur J Endocrinol 2012;166:143-150. 
2. Ahlawat SK, Jain S, Kumari S, et al. Pheochromocytoma associated with pregnancy: Case report and review of the literature. Obstet Gynecol Surv 1999;54:728-737.

3. Pace DE, Chiasson PM, Schlachta CM, et al. Minimally invasive adrenalectomy for pheochromocytoma during pregnancy. Surg Laparosc Endosc Percutan Tech 2002;12:122-125.

4. Donatini G, Kraimps JL, Caillard C, et al. Pheochromocytoma diagnosed during pregnancy: Lessons learned from a series of ten patients. Surg Endosc 2018;32:3890-3900.

Address correspondence to: Rajeev Kumar, MCh Department of Urology All India Institute of Medical Sciences New Delhi India 110029

E-mail: rajeev.urology@aiims.edu

\begin{tabular}{|c|}
\hline Abbreviations Used \\
$\mathrm{CT}=$ computed tomography \\
DOTANOC PET-CT $=[1,4,7,10$-tetraazacyclododecane- \\
$1,4,7,10$-tetraacetic acid $]-1$ - \\
NaI $(3)$-octreotide positron \\
emission tomography-CT \\
$\mathrm{MIBG}=131 \mathrm{I}$-meta-iodobenzylguanidine \\
$\mathrm{MRI}=$ magnetic resonance imaging
\end{tabular}

Cite this article as: Prakash $\mathrm{P}$, Kulshrestha V, Ramachandran R, Kumar R (2020) Successful pregnancy outcomes after laparoscopic management of pheochromocytoma, Journal of Endourology Case Reports 6:3, 170-173, DOI: 10.1089/cren.2019.0139. 